\title{
Recollection of a simpler time
}

\section{The death of Paul Dirac a week ago is a reminder of the precocious and staggering achievement, sixty years ago, of one of the most diffident of contemporary heroes}

THE death of P.A.M. Dirac, in Tallahassee, Florida, on 20 October is a moving reminder of what science was like when everything was much simpler than now. The tale is that, as a shy but clever son of a Swiss-born schoolteacher in Bristol in the West of England, he embarked on a degree course in electrical engineering at the local university, just two months before the First World War ended. With a degree but no job, he took a degree in mathematics, as it were to fill in time. By 1923 , with the help of a scholarship and a government grant, he was a research student at the University of Cambridge. And then, two years later, at the age of 23 , he had put Heisenberg's quantum mechanics, not then published, on an axiomatic foundation that still endures.

How could so much have been done in so short a time? Luck must be a part of any explanation. Dirac was lucky that his Cambridge supervisor was R.H. Fowler, then full of excitement about the statistical mechanics of stellar atmospheres. Dirac was also lucky that Heisenberg went to Cambridge to talk at the Kapitza Club (then Cambridge's private phi beta kappa) in July 1925, and sent Fowler a set of the proofs of his first paper on new quantum theory by the middle of August. Who to ask to read them but Dirac? The real luck, of course, was that the time was ripe for a radical upheaval. Planck quanta were real enough but the old quantum theory of atoms, which worked well enough for atomic hydrogen, was increasingly at odds with spectroscopy.

But Dirac's special contribution to quantum mechanics was its independence of what others were about. Heisenberg's first paper (Zeits.f.Phys. 33, 879-893; $1925)$, the proofs of which Dirac had seen, had set the agenda - how to generalize Bohr's prescription that quantum states are those calculated by classical mechanics which happen to satisfy a quantum condition pulled like a rabbit from a hat. Heisenberg's innovation was to describe the kinematics and the dynamics of quantum variables strictly analogous to the classical dynamical variables - position, momentum and so on.

Dirac's first paper on the subject was received at the Royal Society on 7 November 1925, and published the following month in Proc. Roy. Soc. A 109, 642-653 (which says something else about the simplicity of those times). Its contemporary importance was threefold.
It emphasized that the quantities representing dynamical variables in quantum mechanics are elements in an algebra then undefined, it demonstrated that the circumstance that two variables, say $q$ (for position) and $p$ (for conjugate momentum), do not commute is not an embarrassment (as it had seemed to Heisenberg) but a natural way of making a bridge with the quantities in classical Hamiltonian mechanics called Poisson brackets.

Dirac's achievement in the following five years was staggering. By the time his $\mathrm{PhD}$ thesis had been submitted (in the spring of 1926), he had made a first attempt at the problem of relativistic quantum mechanics. This was the train of argument that led to Dirac's relativistic equation of the electron which provided both a natural, not ad hoc, way of accommodating electron spin and as clear a prediction that positrons must also exist as any more recent prediction of a particle of matter. Almost in passing, Dirac quantized Maxwell's equations, providing the foundations of a quantum theory of radiation and a model for what is now called field theory.

Throughout this revolution, Dirac's style was distinctive. He was not so much an iconoclast as an adventurous spirit, sceptical of fudges but prepared to see where logic would lead. What else can account for his failure to accept as his own the idiom in which his elders and presumptive betters described the problems facing physics in 1925 ?

The point is nicely illustrated by Dirac's own algebra for the dynamical variables of a quantum system. Heisenberg had shown that the quantum condition boils down to the simple relation that $q p-p q=\mathrm{i} h$ if $q$ and $p$ are, respectively a position and its conjugate momentum (with $\mathrm{i}$ the square root of -1 and $h$ Planck's constant divided by $2 \pi$ ). When Born and Jordan had reminded Heisenberg that his quantum equations embodied the rules of matrix multiplication, the three went on to elaborate their doctrine of matrix mechanics.

Not Dirac. The quantum variables were the elements of an algebra whose rules were dictated by the physics. Dirac called them q-numbers (Proc. Roy. Soc. $A$ 110, $561-569 ; 1926)$ and said this about them:

At present one can form no picture of what a q-number is like. One cannot say that one q-number is greater or less than another. All one knows about q-numbers is that if $z_{1}$ and $z_{2}$ are two $q$-numbers... there exist the numbers $z_{1}+z_{3}, z_{1} z_{2}$ and $z_{2} z_{1}$ which will in general be q-numbers... One knows nothing about the way the numbers are formed except that they satisfy all the ordinary laws of algebra, excluding the commutative law of multiplication.

At any point along the way, Dirac could have resolved this uncertainty by changing idiom. Representing q-numbers by matrices would have given Heisenberg's quantum mechanics, representing them by differential operators would have given Schrödinger's wave mechanics. But why needlessly particularize?

The scale of Dirac's achievement during this short spell is now not easily comprehended. It is as if he had found a secret window on the foundations of physics, a companion for that through which Einstein looked in 1905 . It cannot often have been that a physics textbook (The Principles of Quantum Mechanics, Oxford, 1930) should be nothing but a record if its author's research.

Dirac's distinctive style stamps this great book and the generation of students brought up on it - or in its shadow. Dirac himself, however strongly convinced in 1930 that the problems of physics had been solved, seems not to have been filled with proselytizing zeal. Always shy, he seems to have been tragically shy of students. Much of the 1930s were spent in a hunt for some way of ridding calculations of radiation fields of the infinities that bedevilled them. Dirac did not succeed in this herculean task, completed only in 1948 by Schwinger, Tomonaga and Feynmann.

But from this period there survive three novel ideas - the notions that magnetic monopoles may exist, that it cannot be a mere coincidence that dimensionless numbers such as the ratio of electrical to gravitational forces are equal to the age of the Universe (in atomic units) and (as a consequence) that the gravitational constant should decrease with increased age of the Universe (Nature 139, 323; 1937). Time, no doubt, will show what happens to these conjectures.

Dirac's style persists. The anonymous reviewer of the first edition of his book (Nature 127, 699; 1931) said of Dirac that "he bids us throw aside preconceived ideas regarding the nature of phenomena... we may describe this as the application of pure thought to physics". He was a subversive whose pure thought was more penetrating than most other people's. John Maddox 